\title{
Découverte d'un carcinome épidermoïde du trigone rétromolaire chez un jeune adulte sans facteurs de risque
}

\author{
Yordan Benhamou ${ }^{1}{ }^{*}$, Hélène Raybaud ${ }^{1}$, Gilles Poissonnet ${ }^{2}$, Patrice Cochais ${ }^{1}$, \\ Patrick Mahler ${ }^{1}$ \\ 1 Service d'odontologie, $\mathrm{CHU}$ de Nice, France \\ ${ }^{2}$ Service de chirurgie ORL face et cou, Centre Antoine Lacassagne, Nice, France
}

(Reçu le 7 décembre 2013, accepté le 10 avril 2014)

Mots clés : carcinome épidermoïde oral / jeune / facteurs de risque

Key words: oral squamous cell carcinoma (OSCC) / young adult / risk factors
Résumé - Introduction : Le carcinome épidermoïde oral est habituellement diagnostiqué chez des patients alcoolo-tabagiques entre 50 et 60 ans. Des cas de carcinomes épidermoïdes oraux sont de plus en plus rapportés chez des patients jeunes sans facteurs de risques habituels, entrainant une errance diagnostique et un mauvais pronostic. Observation : Le diagnostic tardif d'un carcinome épidermoïde mandibulaire survenu chez un jeune patient de 27 ans sans facteurs de risque est rapporté. En dépit d'un traitement chirurgical, d'une chimiothérapie ciblée, et d'une radiothérapie, le patient n'a pas survécu. Discussion : L'interprétation des informations cliniques pourrait aider à comprendre ce qui provoque l'apparition prématurée de ces cancers et leur agressivité.

\begin{abstract}
Discovery of squamous cell carcinoma of the retromolar trigone in a young adult without risk factors. Introduction: Oral squamous cell carcinoma (OSCC) is usually diagnosed in a context of alcohol and tobacco intoxication in patients between 50 and 60 years of age. Cases of OSCC are increasingly found in young patients without any of the usual risk factors, resulting in diagnostic uncertainty and poor prognosis. Case report: This article reports a case of late diagnosis of mandibular OSCC in a young 27-year-old male patient with no risk factors. Despite surgical treatment combined with targeted chemotherapy and radiotherapy, the patient did not survive. Discussion: Interpretation of clinical data might help in understanding what underlies early development of these cancers and their aggressiveness.
\end{abstract}

\section{Introduction}

Le carcinome épidermoïde est une tumeur maligne ayant pour origine une prolifération incontrôlée des cellules épithéliales. En France, plus de $90 \%$ des cancers des voies aérodigestives supérieures (VADS) sont des carcinomes épidermoïdes [1].

La forme orale est surtout décrite chez des patients masculins alcoolo-tabagiques de plus de 50 ans. Un nombre croissant de cas d'une forme majoritairement localisée au niveau de la langue, chez des sujets jeunes sans facteurs de risque a été rapporté [2,3]. L'étiologie est encore mal connue, certains auteurs ont évoqué une infection par le virus du papillome humain (HPV), non prouvée à ce jour. Cet article rapporte le diagnostic et l'évolution défavorable d'un carcinome épidermoïde rétro-molaire chez un jeune homme sans facteurs de risque. L'objectif est de sensibiliser les chirurgiens-dentistes au diagnostic précoce des cancers oraux des patients dont l'âge ou les facteurs de risque diffèrent du profil habituel.

\section{Observation clinique}

Un jeune homme de 27 ans a consulté les urgences du service d'odontologie du CHU de Nice, pour des douleurs intermittentes au niveau de la $3^{\mathrm{e}}$ molaire mandibulaire droite.

Le questionnaire médical n'a révélé la présence d'aucune pathologie ni traitement médicamenteux au long cours. Le patient ne présentait pas d'habitude toxique alcoolique ou tabagique. L'examen clinique et radiographique évoquait une péricoronarite aiguë de 48 . Le traitement d'urgence a consisté en une prescription d'antibiotiques (amoxicilline $2 \mathrm{~g}$ par jour pendant 6 jours) et d'antalgiques ainsi que des conseils et recommandation d'avulsion en cas de récidive.

\footnotetext{
*Correspondance : yordan.benhamou@gmail.com
} 


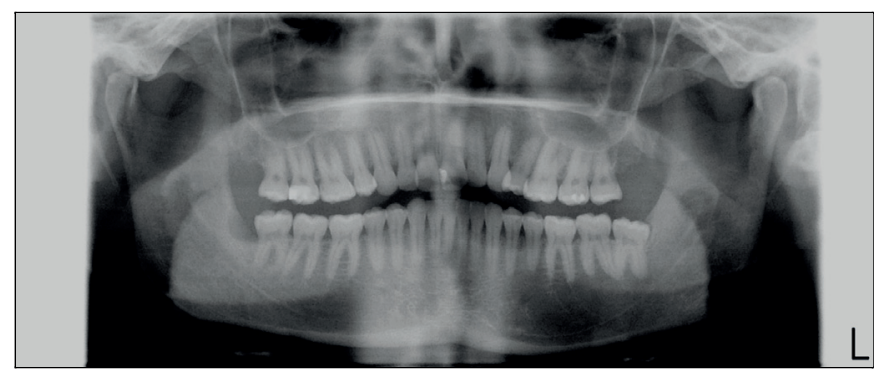

Fig. 1. Orthopantomogramme avant l'avulsion de la dent 48. Observation d'une image radio-claire en demi-lune contre le rebord antérieur de la branche montante de la mandibule.

Fig. 1. Orthopantomogram before avulsion of the tooth 48. Observation of a radiolucent image on the anterior edge of the mandible ramus.

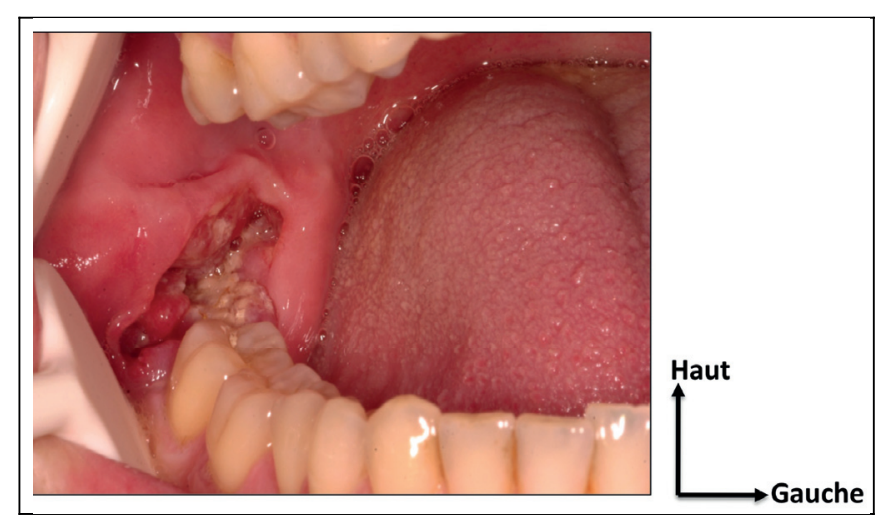

Fig. 2. Lésion ulcéreuse à bords ourlés et à fond kératosique et saigneux ne guérissant pas 1 mois après l'avulsion de la dent 48 .

Fig. 2. Ulcerative lesion with keratosis and without healing 1 month after avulsion of the tooth 48.

Trois mois plus tard, les douleurs sont réapparues, s'accompagnant d'une mobilité de la 48. L'examen d'imagerie montrait une lésion radio-claire arciforme entourant la dent et se prolongeant en distal contre le rebord antérieur de la branche mandibulaire droite (Fig. 1). Le chirurgien-dentiste a procédé à l'avulsion de la 48. Durant les deux semaines suivant cette intervention, le patient a ressenti de vives douleurs. La zone d'avulsion non cicatrisée évoquait une alvéolite aiguë. Un traitement par curetage alvéolaire et pose $d^{\prime}$ Alveolopenga ${ }^{\circledR}$ a été réalisé.

Deux semaines plus tard, le patient a consulté les urgences du service d'odontologie. Le tableau clinique était une absence de cicatrisation du site extractionnel et une persistance des douleurs. L'inspection exobuccale montrait une légère asymétrie faciale. La palpation a révélé la présence d'une adénopathie sous angulomandibulaire indurée et peu mobile de $2 \mathrm{~cm}$ de diamètre. L'examen des territoires d'innervation de la face était normal. L'inspection endobuccale a objectivé une lésion hétérogène bourgeonnante et ulcéreuse à fond saigneux $d^{\prime}$ une dimension de $3,5 \mathrm{~cm}$ de diamètre (Fig. 2). La radiographie

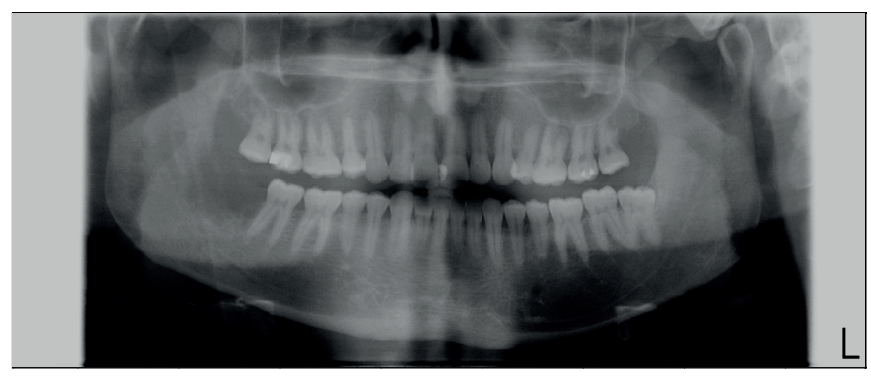

Fig. 3. Orthopantomogramme montrant une image radio-claire en demi-lune mal délimitée de $3 \mathrm{~cm}$ de grand axe, sur le site d'avulsion de la dent 48.

Fig. 3. Orthopantomogram showing a radiolucent image on 48 avulsion site.

panoramique montrait une image radio-claire localisée au niveau de l'angle mandibulaire droit, aux bords mal délimités, en regard de l'alvéole de la 48 (Fig. 3).

Compte tenu de l'aspect carcinomateux de la lésion, et de sa persistance au-delà de deux semaines, une biopsie a été effectuée.

L'analyse histopathologique a révélé la présence d'un carcinome épidermoïde invasif très inflammatoire et bien différencié kératinisant du trigone rétromolaire (Fig. 4). Un immunomarquage du R-EGF a montré une surexpression de ce récepteur alors que la protéine P16 était négative. Un immunomarquage de l'antigène $\mathrm{Ki}-67$ montrait une intense activité mitotique avec $50 \%$ des noyaux marqués. La tumeur était d'emblée classée T4N1Mx selon la classification TNM.

Le patient a été orienté vers le service de chirurgie $0 R L$ face et cou du Centre Antoine Lacassagne. Le bilan d'extension a consisté en un examen TDM (tomodensitométrique) cervicofacial, cérébral et thoraco-abdominal qui montrait la présence de deux adénopathies nécrotiques à droite. Un examen d'imagerie par résonance magnétique avec du gadolinium a permis d'objectiver une lésion de $42 \mathrm{~mm}$ antéropostérieurs $\times 45 \mathrm{~mm}$ dans le sens cranio-caudal $\times 33 \mathrm{~mm}$ dans le sens latéral. Cette lésion volumineuse envahissait en dehors le muscle masséter et en dedans le muscle ptérygoïdien médial. On retrouvait les deux éléments nécrotiques ganglionnaires déjà observés sur le scanner, ce qui classait finalement la tumeur en T4N2bM0.

Le traitement décidé en réunion de concertation pluridisciplinaire a consisté pour la partie chirurgicale en une pelvimandibulectomie interruptrice droite avec désarticulation, un curage ganglionnaire cervicofacial bilatéral et une reconstruction simultanée de l'hémimandibule par transplant osseux vasculaire libre prélevé sur le péroné droit (Fig. 5). Une radiothérapie conformationnelle avec modulation d'intensité à une dose de 60 Gy au centre de la tumeur et sur les aires ganglionnaires a été réalisée. Les tests histologiques ayant révélé la présence du R-EGF en grande quantité, une thérapie ciblée avec un anticorps monoclonal anti-R-EGF (cetuximab) a été instaurée en plus d'une chimiothérapie avec du cisplatine. Les 

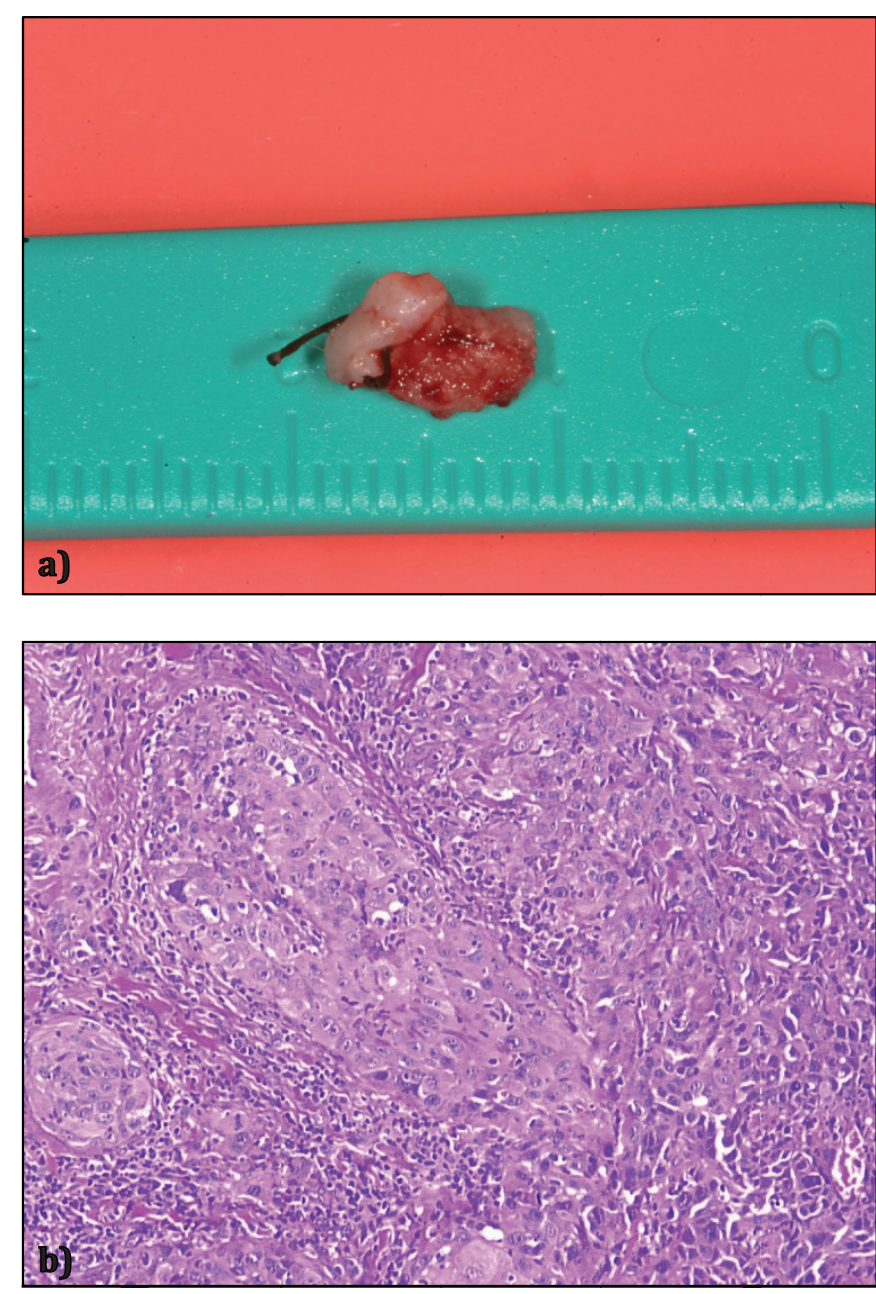

Fig. 4. a) Biopsie au bistouri circulaire, diamètre $5 \mathrm{~mm}$, profondeur $7 \mathrm{~mm}$. b) Examen histopathologique en coloration HES grossissement $100 \times$. Infiltrat inflammatoire important et bourgeons carcinomateux.

Fig. 4. a) Circular scalpel biopsy $5 \mathrm{~mm}$ in diameter and $7 \mathrm{~mm}$ in depth. b) Histopathological examination (HES staining - 100x magnification): inflammatory infiltrate and squamous cell carcinoma.

suites opératoires ont été simples. Dix mois plus tard, des métastases multiples osseuses et pulmonaires ont été découvertes à la scintigraphie et confirmées par la fibroscopie. Malgré de nouvelles séances de radiothérapie et chimiothérapie, le patient est décédé des suites de son cancer 30 mois après sa découverte.

\section{Discussion}

En France, les cancers des VADS (Voies Aéro Digestives Supérieures) se situent au $5^{\mathrm{e}}$ rang des cancers les plus fréquents (plus de 14000 nouveaux cas estimés en 2010) et concernent principalement les hommes ( $75 \%$ des nouveaux cas) [1].

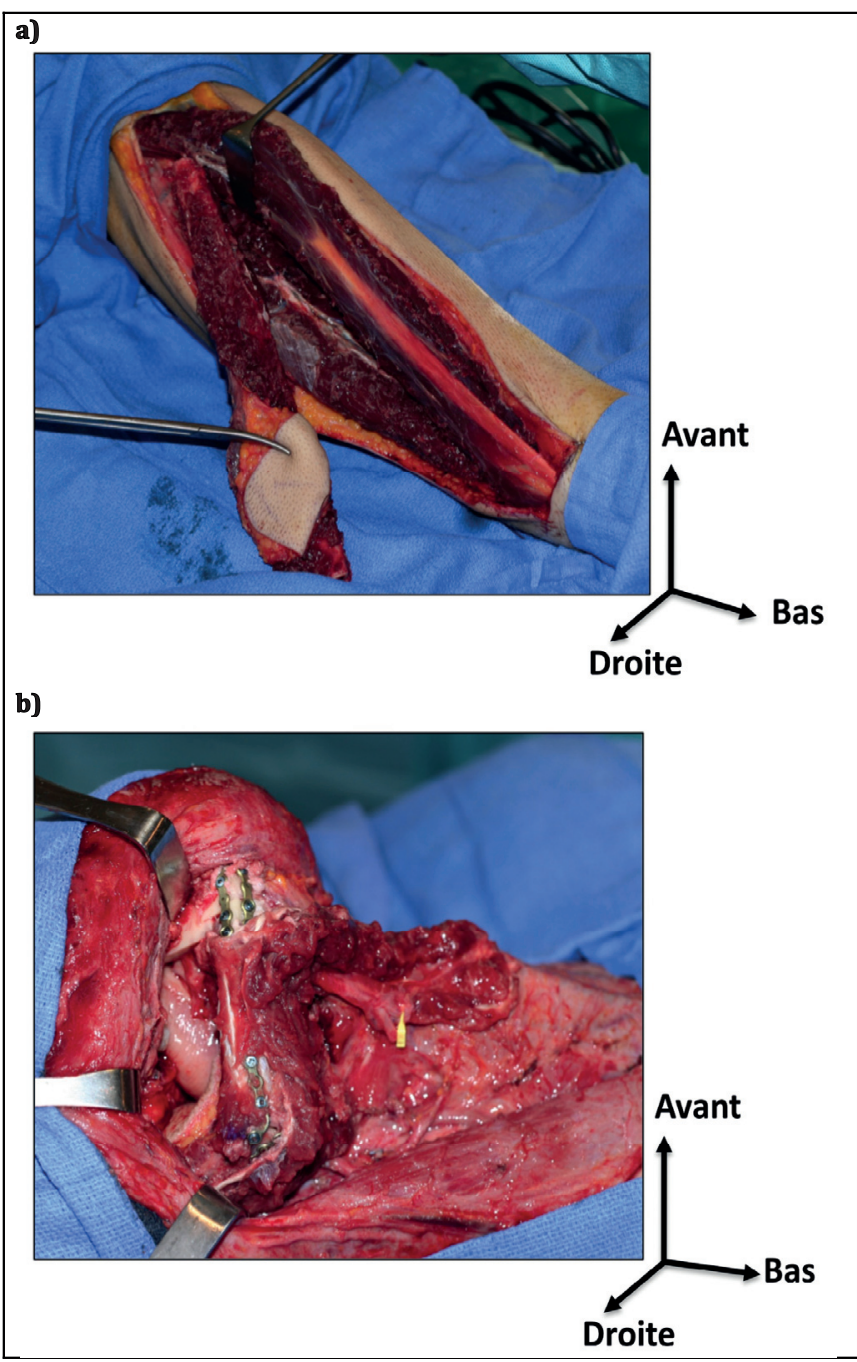

Fig. 5. Chirurgie d'exérèse carcinomateuse. a) Un greffon de péroné droit en cours de prélèvement. b) Fixation du greffon libre après avoir été transformé en hémimandibule avec des plaques d'ostéosynthèse.

Fig. 5. Surgical excision: a) right fibula graft during removal. b) Determination of free graft after being transformed into hemimandible with bone plates.

Le carcinome épidermoïde de l'adulte jeune (moins de 40 ans) semble être une entité à part. En France, en 2012, dans la tranche d'âge de 25-29 ans, la prévalence était de 0,9 femme contre 0,5 homme pour 100000 , toutes localisations orales confondues [4]. Ces observations sont à confronter avec celles d'autres pays dans lesquels le sex-ratio est en défaveur de l'homme. Au niveau clinique, la localisation gingivale devient la plus fréquente [5]. L'évolution clinique se démarque du carcinome épidermoïde de l'adulte avec une tendance à l'invasion métastatique significativement plus élevée même si on ne retrouve pas de différence au niveau des grades histologiques $[5,6]$. Le pronostic est beaucoup plus sombre chez les moins 
de 30 ans avec, en cas de récidive, $100 \%$ de décès à 16 mois [7] contre 12 à $50 \%$ de survie à 5 ans selon les études chez l'adulte plus âgé [8].

L'étiologie diffère également, les facteurs extrinsèques classiques tels que le tabac et l'alcool ne sont pas toujours retrouvés [3,9]. Le seuil d'intoxication (environ 21 années de tabagisme) n'est jamais atteint chez le jeune car l'exposition est trop courte. Certains auteurs suggèrent qu'une augmentation des rapports sexuels oro-génitaux chez les jeunes serait à l'origine d'une contamination HPV, augmentant ainsi le risque de carcinome oral [10]. Aucune infection par HPV n'a été retrouvée dans le cas présenté.

Pour tenter d'expliquer la survenue de ces cancers chez le jeune, certains auteurs ont suggéré une instabilité génétique plus importante. Les cellules tumorales comprennent les mêmes altérations de l'ADN que l'adulte plus âgé au niveau des séquences microsatellites [11] avec notamment une mutation du gène codant pour la protéine P53 appelée « gardienne du génome ». Cette mutation a été retrouvée dans $45 \%$ des cancers d'adultes jeunes [12]. Cette vision unifactorielle est aujourd'hui dépassée et la littérature parle d'un ensemble $d^{\prime}$ anomalies ayant un effet modificateur [13].

Il est actuellement admis que le carcinome épidermoïde oral est surtout lié à nombre de dérèglements en aval de la cascade d'activation du récepteur R-EGF aboutissant à une prolifération incontrôlée des cellules et à un échappement aux mécanismes d'apoptose [14-16]. La vitesse de renouvellement cellulaire associée à une surexpression et une hyperactivité du récepteur R-EGF se traduit par une taille de tumeur plus importante. Le marquage de la protéine Ki-67 a permis de confirmer l'intense activité mitotique dans la biopsie du cas clinique présenté. L'issue fatale en dépit des traitements pourrait être liée à l'hétérogénéité intratumorale $[17,18]$ démontrée dans de nombreux cancers. Le tissu tumoral étant constitué de différents clones cellulaires carcinomateux, les traitements par chimiothérapie anti R-EGF (cetuximab) et radiothérapie combinée n'ont fait qu'éliminer certains clones, sélectionnant les cellules les plus résistantes ou indifférenciées de la tumeur. Ces cellules ont ensuite proliféré à bas bruit et se sont différenciées (période de rémission transitoire) jusqu'à devenir des tumeurs récidivantes lorsque leur masse est devenue cliniquement détectable à dix mois. Le contrôle impossible des métastases a été fatal au patient.

Deux cas de cancers de la cavité buccale chez des jeunes ont été publiés en France, ce qui souligne la rareté des cas [19].

\section{Conclusion}

D'incidence extrêmement rare chez des adultes jeunes sans facteurs de risque associés, le diagnostic de carcinome épidermoïde oral est souvent tardif, exposant le malade à des traitements lourds et à un risque de décès accru. Le manque d'outils diagnostiques, thérapeutiques et prédictifs dans le pronostic de ces cancers ouvre la voie à de nouvelles investigations comme l'établissement d'un interactome pour connaître les protéines intervenant dans leur évolution clinique.

Une identification des sous-types de carcinomes épidermoïdes oraux pourrait apporter une explication aux différences constatées entre les patients jeunes et les patients âgés. Un examen clinique locorégional complet ainsi qu'une biopsie doivent être réalisés lorsqu'il y a un doute sur une lésion persistant plus de deux semaines.

\section{Conflits d'intérêt : aucun}

\section{Références}

1. HAS InCA. La prise en charge du cancer des voies aéro-digestives supérieures [Internet]. [cité 4 mars 2014]. Disponible sur: http://www.e-cancer.fr/component/docman/doc_download/ 9368-guide-patient-affection-de-longue-duree-la-prise-encharge-du-cancer-des-voies-aerodigestives-superieures

2. Van Monsjou HS, Wreesmann VB, Van den Brekel MWM, Balm AJM. Head and neck squamous cell carcinoma in young patients. Oral Oncol 2013;49(12):1097-1102.

3. Llewellyn C, Johnson N, Warnakulasuriya KAA. Risk factors for squamous cell carcinoma of the oral cavity in young people - a comprehensive literature review. Oral Oncol 2001;37(5): 401-418.

4. Ligier $\mathrm{K}$, et al. [Epidemiology of oral cavity cancers in France]. Rev Stomatol Chir Maxillofac 2011;112(3):164-171.

5. Acharya S, Tayaar AS. Analysis of clinical and histopathological profiles of oral squamous cell carcinoma in young Indian adults: A retrospective study. J Dent Sci 2012;7(3):224-230.

6. Ribeiro ACP, Silva ARS, Simonato LE, Salzedas LMP, Sundefeld MLMM, Soubhia AMP. Clinical and histopathological analysis of oral squamous cell carcinoma in young people: A descriptive study in Brazilians. Br J Oral Maxillofac Surg 2009;47(2):95-98.

7. Hilly 0 , et al. Carcinoma of the oral tongue in patients younger than 30 years: Comparison with patients older than 60 years. Oral Oncol 2013;49(10):987-990.

8. De Raucourt D, Rame J-P, Louis M-Y. [Principal treatments of head and neck cancer]. Rev Prat 2006;56(15):1662-1666.

9. Falaki $F$, et al. Clinical and histopathological analysis of oral squamous cell carcinoma of young patients in Mashhad, Iran: a retrospective study and review of literature. Med Oral Patol Oral Cir Bucal 2011;16(4):e473-477.

10. Nelke KH, Lysenko L, Leszczyszyn J, Gerber H. Human papillomavirus and its influence on head and neck cancer predisposition. Postepy Hig Med Dosw (Online) 2013;67: 610-616.

11. Jin Y-T, Myers J, Tsai S-T, Goepfert H, Batsakis J, El-Naggar A. Genetic alterations in oral squamous cell carcinoma of young adults. Oral Oncol 1999;35(3):251-256.

12. Braakhuis $B$, et al. TP53 mutation and human papilloma virus status of oral squamous cell carcinomas in young adult patients. Oral Dis 2013. 
13. Bernstein JM, Bernstein CR, West CML, Homer JJ. Molecular and cellular processes underlying the hallmarks of head and neck cancer. Eur Arch Otorhinolaryngol 2013;270(10):2585-2593.

14. Chin D, Boyle GM, Theile DR, Parsons PG, Coman WB. Molecular introduction to head and neck cancer (HNSCC) carcinogenesis. $\mathrm{Br}$ J Plastic Surg 2004;57(7):595-602.

15. Jeon GA, et al. Global gene expression profiles of human head and neck squamous carcinoma cell lines. Int J Cancer 2004;112(2): 249-258.
16. Chen Y-J, et al. Genome-wide profiling of oral squamous cell carcinoma. J Pathol 2004;204(3):326-332.

17. Marusyk A, Almendro V, Polyak K. Intra-tumour heterogeneity: a looking glass for cancer? Nat Rev Cancer 2012;12(5):323-334.

18. Greaves M, Maley CC. Clonal evolution in cancer. Nature 2012; 481(7381):306-313.

19. D'Elbée J-M, Ella B, Fricain J-C. Carcinome épidermoïde buccal chez le sujet jeune : présentation d'un cas. Médecine Buccale Chirurgie Buccale 2012;19(1):39-43. 